\title{
Review of: "Retentive capacity of power output and linear versus non-linear mapping of power loss in the isotonic muscular endurance test"
}

\author{
Hamed Esmaili
}

Potential competing interests: The author(s) declared that no potential competing interests exist.

Manuscript entitled "Retentive capacity of power output and linear versus non-linear mapping of power loss in the isotonic muscular endurance test" specially measuring power output in fatigued state. This study showed that Muscular endurance required in real-world activities can be measured by considering the amount of work produced or the activity duration via the recommended IME testing protocol under a psychophysiological termination criterion. Non-linear mapping techniques provide more powerful mapping of power loss compared with linear mapping in the IME testing protocol. The topic is too interesting in muscle function area and provides in depth information about muscle power output in fatigued condition. In my opinion the authors must be encouraged. However, there is some concerns that must be noted before publishing in this journal. The concerns are listed below:

Introduction

1. Provide some evidences related to changes in EMG activity of muscles in fatigued state in introductory section.

2. How EMG could be affected by power output? The link between EMG activity and power output is missing in the introduction section.

Methods

1. Why you have considered only right feet?

2. Provide references for your protocol.

3. Provide some information about electrodes placement.

4. Your participants showed some degrees of divergency in repetition of plantar flexion and other tasks. This means difference in muscular endurance among your participants. It could be source of some other influential factors. How could you control the effect of this divergency?

Discussion

1. There is irrelevant sentences is this section. Most of them could be placed in introductory section. It is recommended to critically review this section. 